\title{
Reoptimization of the Minimum Total Flow-Time Scheduling Problem
}

\author{
Guy Baram and Tami Tamir \\ School of Computer Science, The Interdisciplinary Center, Herzliya, Israel \\ guy.baram@gmail.com, tami@idc.ac.il
}

\begin{abstract}
We consider reoptimization problems arising in production planning. Due to unexpected changes in the environment (out-of-order or new machines, modified jobs' processing requirements, etc.), the production schedule needs to be modified. That is, jobs might be migrated from their current machine to a different one. Migrations are associated with a cost - due to relocation overhead and machine set-up times. The goal is to find a good modified schedule, which is as close as possible to the initial one. We consider the objective of minimizing the total flow time, denoted in standard scheduling notation by $P \| \sum C_{j}$.

We study two different problems: $(i)$ achieving an optimal solution using the minimal possible transition cost, and (ii) achieving the best possible schedule using a given limited budget for the transition. We present optimal algorithms for the first problem and for several classes of instances for the second problem.
\end{abstract}

\section{Introduction}

This work studies a reoptimization variant of the classical scheduling problem of minimizing the total flow time (denoted in standard scheduling notation by $P \| \sum C_{j}$ [12]). This problem can be solved efficiently by the simple greedy SPT rule 23:9] that assigns the jobs in nondecreasing order by their length. This algorithm, as many other algorithms for combinatorial optimization problems, solves the problem from scratch, for a single arbitrary instance without having any constraints or preferences regarding the required solution - as long as it achieves the optimal objective value. However, many of the real-life scenarios motivating these problems involve systems that change dynamically over time. Thus, throughout the continuous operation of such a system, it is required to compute solutions for new problem instances, derived from previous instances.

Moreover, since there is some cost associated with the transition from one solution to another, a natural goal is to have the solution for the new instance close to the original one (under certain distance measure). Thus, solving a reoptimization problem combines the challenge of computing an optimal (or close to the optimal) solution for the new instance, with the challenge of efficiently converting the initial solution to the new one. Each of these challenges, even when considered alone, gives rise to many theoretical and practical questions. 
Obviously, combining the two challenges is an important goal, which naturally shows up in many applications.

Reoptimization variants of scheduling problems arise naturally in production planning - due to unexpected changes in the environment (out-of-order or new machines, modified jobs' processing requirements, etc.). Migrating tasks among the machines is costly, due to relocation overhead and machine set-up times. This work studies the problem of finding a good modified schedule, which is as close as possible to the initial one. To the best of our knowledge, no previous work combines these two objectives in a scheduling setting.

Applications: As mentioned above, the scenario we consider often arises in manufacturing systems. In fact, our work is relevant to any dynamic scheduling environment. We describe below two less intuitive applications in cloud computing and semiconductor wafers production line.

Consider an RPC (Remote Procedure Call) service. In this environment, a cloud of servers can provide service to a limited number of simultaneous users. If the number of requests is high, another virtual server could be temporarily rented, where the cost for using it is per user. The options are to put the RPC in a queue, thus causing latency in the service, or renting more virtual servers, enabling faster service and paying the additional servers' cost. In this application, the transition cost is not due to the migration itself, but due to the activation cost of the additional resources.

Some of our results will be extended to consider modifications that occur after the processing has begun, that is, at time $t>0$. For this extension (see Section 2.1.1) we distinguish between environments in which the currently processed jobs can migrate and be restarted on a different machine, and applications in with restarts are not allowed, and a currently processed job must complete its partial processing. The following application describes a system in which restarts are not allowed: In a semiconductor wafers production line, some of the coating methods involve purely physical processes such as high temperature vacuum evaporation (physical vapor deposition - PVD). During the process, a vacuum is created to enable the coating. Once the elements are in a vacuum environment, the process can not be stopped as if the machine halts, it will be severely damaged [16]. Assume that at time $t>0$ machines are added. Transferring jobs is costly - to capture the transition overhead and the changes required in programming the machines workplan. Also, the elements that are currently produced, that are already in vacuum state, must complete their production.

\subsection{Problem Statement and Notation}

An instance of our problem consists of a set $J_{0}$ of $n_{0}$ jobs and a set of $m_{0}$ identical machines. Denote by $p_{j}$ the processing time of job $j$. A schedule $S_{0}$ of the initial instance is given. That is, for every job in $J_{0}$, it is specified on which machine it is assigned and on which time interval it is going to be processed. At any time, a machine can process at most one job and a job can be processed by at most one machine. 
At time $t \geq 0$, a change in the system occurs. Possible changes include addition or removal of machines and/or jobs, as well as modification of processing time of jobs in $J_{0}$. Let $J$ denote the modified set of jobs, and let $n=|J|$. Let $M$ denote the modified set of machines, and let $m=|M|$. Our goal is to suggest a new schedule, $S$, for the modified instance, with good objective value and small transition cost form $S_{0}$. Assignment of a job to a different machine in $S_{0}$ and $S$ is denoted migration and is associated with a cost. Formally, we are given a price list $\theta_{i i^{\prime} j}$, such that it $\operatorname{costs} \theta_{i i^{\prime} j}$ to migrate job $j$ from machine $i$ to machine $i^{\prime}$. We consider two problems:

1. Rescheduling to an optimal schedule using the minimal possible transition cost.

2. Given a budget $B$, find the best possible modified schedule that be be achieved without exceeding the budget $B$.

Some of our results assume identical transition costs, that is, for all $j$ and $i \neq i^{\prime}$, $\theta_{i i^{\prime} j}=111$ For a given schedule, let $C_{j}$ be the completion time of job $j$, that is, the time when the process of $j$ completes.

Example: Assume that six jobs of lengths $1, \ldots, 6$ are scheduled on a single machine in an optimal SPT order. Assume that a second machine is added, and that all migrations have unit transition cost. Figure 1(a) presents an optimal modified schedule, for which the total flow-time is $\sum C_{j}=34$. The budget required to reach this schedule (or any other schedule with $\sum C_{j}=34$ ) is 3 . For a given budget, $B=2$, it is possible to move, for example, to the modified schedules given in Figures 1(b) and (c), having total flow-time 36 and 35, respectively. The schedule (c) is optimal for this budget. Note that the natural greedy approach of migrating the long jobs if the budget is low (as in schedule (b)) is sub-optimal. Two other natural approaches of prefix-SPT, or suffix-SPT (use the budget to maximize the prefix of the schedule or the suffix of the schedule that is identical to an SPT schedule) are also sub-optimal 2

$\mathrm{S}_{0}: \quad \mathrm{M}_{1}$\begin{tabular}{|l|l|l|l|l|l|}
\hline & 2 & 3 & 4 & 5 & 6 \\
\hline
\end{tabular}

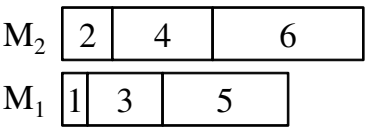

(a)

\begin{tabular}{l|l|l|l|l|}
\cline { 2 - 4 }$M_{2}$ & \multicolumn{2}{|c|}{5} & \multicolumn{1}{c|}{6} \\
\cline { 2 - 5 }$M_{1}$ & 1 & 2 & 3 & 4 \\
\cline { 2 - 4 }
\end{tabular}

(b)

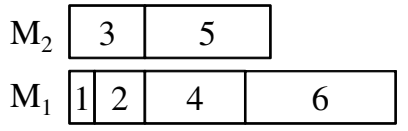

(c)

Fig. 1. An initial assignment (top), an optimal reassignment achieved with transition cost 3 (a), a possible (b) and an optimal (c) reassignments achieved with budget 2

\footnotetext{
1 Note that the constant 1 can be replaced by any other constant.

${ }^{2}$ In the example, schedule (c) is suffix-SPT and optimal, however, suffix-SPT fail on other instances.
} 


\subsection{Related Work}

The 'single-shot' minimum total flow-time, $P \| \sum C_{j}$, can be solved in polynomial time by using the shortest processing time (SPT) rule 2319. The problem is solvable also on unrelated machines, $R \| \sum C_{j}$, 7]14 by a reduction to a minweight complete matching problem.

The work on reoptimization problems started with the analysis of dynamic graph problems (see e.g. 10|24]). Reoptimization algorithms were developed also for some classic problems on graphs, such as shortest-path [1817] and minimum spanning tree [1]. A different line of research deals with the computation of a good solution for an NP-hard problem, given an optimal solution for a close instance. Among the problems studied in this setting are TSP, 446], Steiner Tree on weighted graphs 11 and Knapsack 22. A survey of other research in this direction is given in 3. In all of the above works, the goal is to compute an optimal (or approximate) solution for the modified instance. The resulting solution may be significantly different from the original one, since there is no cost associated with the transition among solutions.

The paper 21] suggests the framework we adopt for this work, in which the solution for the modified instance is evaluated also with respect to its difference from the initial solution. This framework is in use also in [20, to analyze algorithms for data placement in storage area network. Considering both the quality of the solution and the transition cost from an initial solution can also be seen as a special case of multiobjective optimization problems. In these problems, there are several weight functions associated with the input elements. The quality of a solution is measured with respect to a combination of these weights (see e.g., [19[13]).

\subsection{Our Results}

In Section 2 we explore the problem of moving to a modified optimal schedule using the minimal required budget. We present optimal algorithms that return both an optimal schedule and the minimum budget $B$ required to reach an optimal schedule. We first describe an optimal algorithm for arbitrary migration costs and arbitrary changes in the instance. Its running time is dominated by the time required to find a minimum weight complete matching in a complete bipartite graph with $O(\mathrm{~nm})$ vertices. We then present a more efficient algorithm for instances with uniform migration costs. The time complexity of this algorithm is varies between $O(n)$ (if the initial schedule is an SPT schedule) and $O(n \log n)$ (for arbitrary initial schedule). The first algorithm is described assuming the modification takes place at time $t=0$. In Section 2.1.1 we describe how and under which conditions it can be extended to handle modifications at time $t>0$. The second algorithm is valid for changes at any time $t \geq 0$.

In section 3 we consider the problem of rescheduling with a limited budget. The goal is to utilize the budget in the best possible way, that is, the modified schedule should have a low total flow-time - the minimal possible among all schedules that can be achieved using the given budget. Our results for this model 
assume unit migration costs, thus, the budget $B$ gives the maximal number of allowed migrations. We present optimal algorithms for two cases: when the budget is a constant and when migrations are allowed only to new machines.

We conclude, in Section 4, with a discussion and some directions for future work. We note that our results can be applied also on a sequence of modifications. That is, the environment might change more than once, and the algorithms are performed after each modification. Due to space constraints, some of the proofs are omitted. All proofs are available in the full version [5].

\section{Optimal Modified Schedule Using Minimum Budget}

In this section we consider the problem of moving to a modified optimal schedule with respect to the minimal total flow objective using the minimal required budget.

\subsection{Arbitrary Costs and Modifications}

Let $S_{0}$ be a given initial schedule. We do not assume that $S_{0}$ is optimal nor that it has a specific structure or properties. Assume that at time $t=0$, the environment is modified. Possible modifications include addition or removal of machines and/or jobs, and changes in jobs' processing times. The price list $\theta_{i i^{\prime} j}$ specifies for every job $j$ assigned to machine $i$, how much it costs to migrate $j$ to machine $i^{\prime}$. The goal is to find a new schedule, $S$, that is optimal with respect to the total flow-time, and has the minimal transition cost from $S_{0}$ among all optimal schedules.

We reduce the problem into a minimum weight complete matching problem in a bipartite graph. This approach was used by Horn [14, and Bruno, Coffman and Sethi [7] for solving the problem of minimum flow time on unrelated machines $\left(R \| \sum C_{j}\right)$. While the processing time of the jobs do not change due to migrations, it is possible to adopt this technique for our problem by setting the weights in the corresponding bipartite graph in a way that reflects the migration overhead.

Recall that $n$ and $m$ represent the number of jobs and machines in the modified instance. Let $G=(V, E)$, where $V=J \cup U$. The set $J$ represents the set of $n$ jobs (a single node per job). The set $U$ consists of $m n$ nodes, $q_{i k}$, for $i=1, \ldots, m$ and $k=1, \ldots, n$, where node $q_{i k}$ represents the $k^{t h}$ from last position on machine $i$. The edge set $E$ includes an edge $\left(v_{j}, q_{i k}\right)$ for every node in $J$ and every node in $U$ (a complete bipartite graph). The following is an optimal algorithm for our problem. Note that edge weights (determined in Steps 1-2) consist of two components: first, a dominant component corresponding to the contribution of a job assigned in a specific position to the total flow-time, and second, a minor component corresponding to the associated transition cost. Both components are combined to form a single weight. Figure 2 illustrates the edges corresponding to a single job. 
$\underline{\text { Algorithm 1. An optimal algorithms for rescheduling using minimum budget }}$

1. Let $\theta_{i i^{\prime} j}$ be a price list, i.e., it $\operatorname{costs} \theta_{i i^{\prime} j}$ to migrate job $j$ from machine $i$ to machine $i^{\prime}$. In particular, for all $i, j, \theta_{i i j}=0$.

Let $\Delta=\max _{j, i, i^{\prime}} \theta_{i i^{\prime} j}$ and let $Z$ be a constant lager than $n \Delta$.

2. Let $G$ be the complete bipartite graph corresponding to the problem. Set the edge weights as follows:

- For every job that is assigned to $i$, the weight of $\left(v_{j}, q_{i k}\right)$ is $Z k p_{j}$.

- For every $i^{\prime} \neq i$, the weight of $\left(v_{j}, q_{i^{\prime} k}\right)$ is $Z k p_{j}+\theta_{i i^{\prime} j}$.

3. Find a min-cost complete matching in $G$. Let $H$ denote the set of edges in this matching.

4. Return the schedule corresponding to $H$. That is, for every $\left(v_{j}, q_{i^{\prime}, k}\right) \in H$, assign $j$ in the $k^{\text {th }}$ from last position on machine $M_{i^{\prime}}$. The minimum transition cost is $\sum_{\left(v_{j}, q_{i^{\prime}, k}\right) \in H} \theta_{i i^{\prime} j}$, where $i$ is the machine on which $j$ is assigned in $S_{0}$.

In the following claims we show that $H$ induces an optimal schedule with the minimal possible transition cost from $S_{0}$. First, we show that $H$ corresponds to a schedule with minimum total flow-time, then we show that among all schedules achieving minimum total flow-time, the schedule induced by $H$ has minimum transition cost from $S_{0}$.

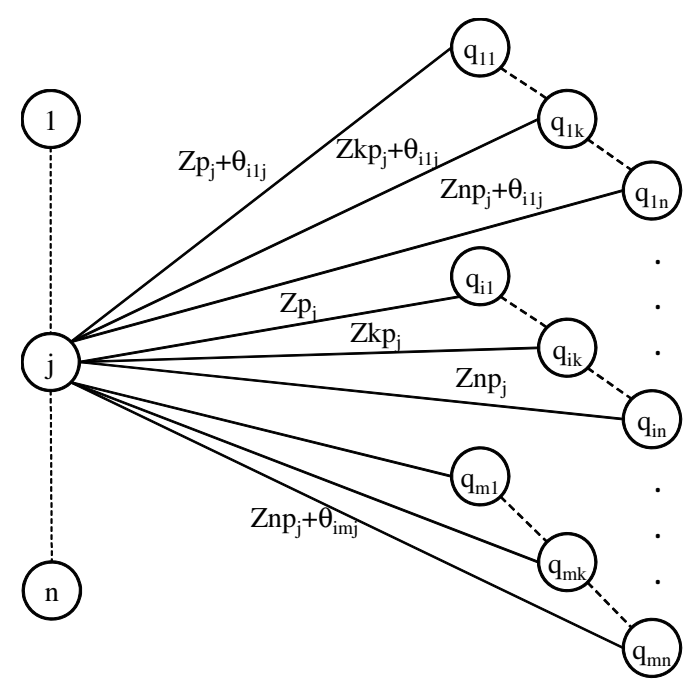

Fig. 2. The bipartite graph for Algorithm 1 The job $j$ is assigned to machine $i$ in $S_{0}$.

Claim. The set of edges $H$ corresponds to a feasible schedule with minimum total flow-time.

Claim. Among all schedules achieving minimum total flow, the schedule induced by $H$ has the minimal transition cost. 
Proof. Let $H^{*}$ be any perfect matching in $G$, corresponding to a schedule, $S\left(H^{*}\right)$, achieving minimum total flow-time. We show that the transition cost to $S\left(H^{*}\right)$ is not lower than the transition cost to $S(H)$. We know that $H$ is a min-cost matching in $G$, therefore, $\sum_{e \in H} w(e) \leq \sum_{e \in H^{*}} w(e)$. Also, since both achieve minimum total flow-time and the weights $w^{\prime}$ reflect the total flow-time without the transition costs, $\sum_{e \in H} w^{\prime}(e)=\sum_{e \in H^{*}} w^{\prime}(e)$. The definition of $w$ implies that for every matching $H^{\prime}$, it holds that $\sum_{e \in H^{\prime}} w(e)=$ $Z \sum_{e \in H^{\prime}} w^{\prime}(e)+\sum_{e=\left(v_{j}, q_{i k}\right) \in H^{\prime}} \theta_{i i^{\prime} j}$, where the second term is exactly the transition cost from the initial schedule to the schedule induced by $H^{\prime}$. We conclude that the transition cost to $S\left(H^{*}\right)$ is not lower than the transition cost to $S(H)$.

\subsubsection{Extension: When the Modification Occurs at Time $t>0$}

The change in the system might occur after the processing has begun, that is, at time $t>0$. Let $J_{t}$ be the set of jobs processed at time $t$. In some systems the processing of $j \in J_{t}$ must complete on its current machine. In others, $j$ can be migrated to another machine. If reassigned, the corresponding transition cost is applied and the job must restart. We assume that preemptions are not allowed 3 . For every machine $i$, let $\gamma_{i}$ denote the time required to complete the job from $J_{t}$ processed at time $t$ on machine $i$.

When restarts are not allowed, the only modification we consider is machines addition. Note that if machines can be removed, and restarts are not allowed then the problem is not well-defined for the jobs that are currently processed. The problem can be viewed as a scheduling problem in which machine $i$ is available starting at time $\gamma_{i}$. Algorithm 1 can be generalized by setting the weights in the bipartite graph (described in Section 2.1) in the following way:

- For every job that is assigned to $M_{i}$, the weight of $\left(v_{j}, q_{i k}\right)$ is $Z\left(k p_{j}+\gamma_{i}\right)$.

- For every $i^{\prime} \neq i$, the weight of $\left(v_{j}, q_{i^{\prime} k}\right)$ is $Z\left(k p_{j}+\gamma_{i^{\prime}}\right)+\theta_{i i^{\prime} j}$.

When restarts are allowed, for every job $j \in J_{t}$ an additional possibility is to migrate $j$ to a different machine and restart its processing. For this case our extension assumes that the initial schedule was optimal, that is, in SPT order. We set the weights in the bipartite graph as follows:

- For every job $j \in J_{t}$ that is currently processed on $M_{i}$ the weight of $\left(v_{j}, q_{i k}\right)$ is $Z k \gamma_{i}$.

- For every job $j \notin J_{t}$ that is assigned to $M_{i}$, the weight of $\left(v_{j}, q_{i k}\right)$ is $Z k p_{j}$.

- For every $i^{\prime} \neq i$, the weight of $\left(v_{j}, q_{i^{\prime} k}\right)$ is $Z k p_{j}+\theta_{i i^{\prime} j}$.

Note that the above weights correspond to the contribution of jobs to the total flow-time, assuming the following property: if a currently processed job $j$ remains on $M_{i}$ then in the optimal modified assignment it is processed first on $M_{i}$. The proof of this property and the extensions' proofs are given in the full version [5].

3 Enabling preemptions affects all the jobs of the instance, thus causing the problem to be intractable 22 . 


\subsection{An Efficient Algorithm for Identical Migration Costs}

In this section we consider systems with identical migration costs, that is, for all $j, i, i^{\prime}$, it holds that $\theta_{j, i, i^{\prime}}=1$. We present an efficient algorithm for finding an optimal modified schedule using the minimal possible budget. The algorithm can be applied for addition or removal of machines and/or jobs, as well as changes in jobs' processing times.

The algorithm is based on some properties of the SPT algorithm [23|9] for $P \| \sum C_{j}$. For completeness, we describe a specific form of SPT algorithm: Given an instance of $n$ jobs and $m$ parallel machines, add dummy jobs of length 0 such that the total number of jobs is a multiple of $m$. Specifically, if $n$ is not a multiple of $m$, then add to the instance $m-(n \bmod m)$ jobs of length 0 . The dummy jobs can be scheduled on arbitrary machines and (when rescheduling) their migration cost is 0 . Given that $n$ is a multiple of $m$, the SPT algorithm can be described as follows: First, sort the jobs in non-decreasing order of processing time (break ties arbitrarily). Next, partition the jobs into $n / m$ rounds of $m$ jobs each. The $k$-th round consists of the jobs indexed $(k-1) m+1, \ldots, k m$ in the sorted list. Schedule on each machine one job from the first round, followed by one job from the second round, etc.

We use the following known property of SPT schedules: the internal assignment of jobs from a particular round to the machines does not affect the total flow-time. That is, any schedule in which the $m$ jobs of round $k$ are assigned on the $k$-th slots of the $m$ machines is optimal.

Let $L$ be the set of job lengths in the modified instance. The set $L$ includes at most $n$ distinct values. By the above property of SPT schedules, an optimal schedule can be characterized by the numbers $n_{\ell, k}$, for all $\ell \in L$ and $1 \leq k \leq \frac{n}{m}$, where $n_{\ell, k}$ is the number of jobs of length $\ell$ in round $k$, in any optimal schedule. Moreover, the problem of finding an optimal schedule using minimum transition cost reduces to the problem of finding a schedule obeying the optimal $n_{\ell, k}$ values with a minimal number of migrations from the initial schedule. The following is an overview of our optimal algorithm:

$\overline{\text { Algorithm 2. An efficient optimal algorithm for rescheduling with identical }}$ migration costs.

1. For every length $\ell \in L$ and round $1 \leq k \leq \frac{n}{m}$, calculate $n_{\ell, k}$, the number of jobs of length $\ell$ in round $k$, in any optimal modified schedule.

2. Partition $L$ into two sets of job lengths: Let $L_{1} \subseteq L$ be the set of lengths such that $\ell \in L_{1}$ if and only if $n_{\ell, k}>0$ for a single round $k$. Let $L_{2}=L \backslash L_{1}$ be the set of lengths such that $\ell \in L_{2}$ if and only if $n_{\ell, k}>0$ for more than a single round.

3. For every round $1 \leq k \leq \frac{n}{m}$, schedule a maximal number of non-migrating jobs in round $k$. First, assign jobs having lengths in $L_{1}$, then in $L_{2}$. When assigning jobs from $L_{2}$, give higher priority to short jobs.

4. Schedule migrating jobs. 
The idea is to assign first a maximal number of non-migrating jobs, and then assign the migrating jobs. When assigning the non-migrating jobs, we first assign the more restricted jobs - having lengths in $L_{1}$, and then the more flexible jobs whose lengths are in $L_{2}$.

Denote by $S$ the schedule built by the algorithm. Steps (3-4) are implemented as follows: Denote by $S_{i, k}$ the slot in the $k^{t h}$ round on machine $i$. Initially, for all $1 \leq i \leq m, 1 \leq k \leq \frac{n}{m}$ it holds that $S_{i, k}$ is available (=EMPTY). During steps (3-4) some slots are assigned to non-migrating jobs. Whenever a job $j$ of length $\ell$ is assigned to the $k$-th slot on machine $i$, the corresponding variable $S_{i, k}$ is set to $j$, and the corresponding counter of $n_{\ell, k}$ is reduced by one. Specifically, steps (3-4) are implemented as follows:

Step 3: Step 3 consists of $\frac{n}{m}$ iterations. In iteration $k$, the algorithm assigns non-migrating jobs into slots of round $k$. Consider a slot $S_{i, k}$. Let ForFree $(i, k)$ denote the set of jobs that can be assigned to $S_{i, k}$ with no migration. Formally, $j \in \operatorname{ForFree}(i, k)$ if and only if $(i) n_{p_{j}, k}>0,(i i) j$ is assigned to $M_{i}$ in $S_{0}$, and (iii) $j$ was not assigned to $M_{i}$ in earlier rounds.

In step 3, if possible, the algorithm assigns to $S_{i, k}$ a job from ForFree $(i, k)$ giving priority to lengths in $L_{1}$, and then to shorter lengths in $L_{2}$. Formally,

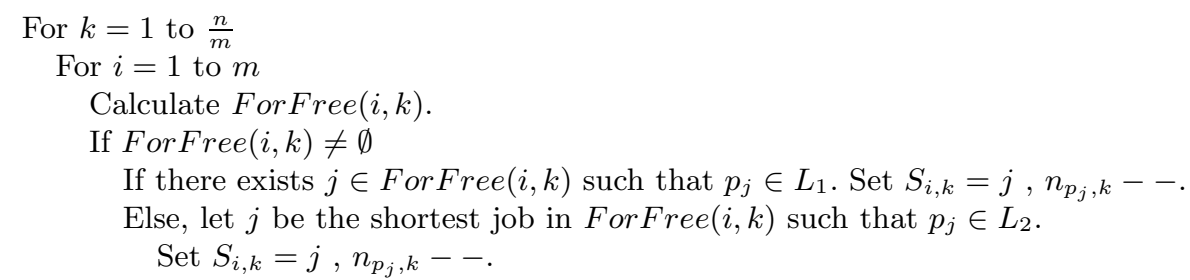

Step 4: Step 4 consists of $\frac{n}{m}$ iterations. In iteration $k$, the algorithm assigns, with migrations, jobs to slots $S_{i, k}$ for which $\operatorname{ForFree}(i, k)=\emptyset$. Formally,

While there exist $\ell, k$ such that $n_{\ell, k}>0$,

Assign any unassigned job $j$ of length $\ell$ to any machine $i$ s.t. $S_{i, k}=E M P T Y$.

Set $S_{i, k}=j, n_{\ell, k}--$.

The number of migrations is the number of non-dummy jobs assigned in step 4 . This number is the minimum budget required to reach an optimal schedule. We prove the optimality of the algorithm by combining two lemmas.

Lemma 1. The algorithm produces an optimal schedule with respect to the total flow-time.

Proof. The schedule $S$ satisfies the $n_{\ell, k}$ values calculated by SPT algorithm, therefore it must be optimal. Since these values were calculated according to the amounts of jobs in the modified instance, all jobs are assigned, that is, in Step 4, while there exist $\ell, k$ such that $n_{\ell, k}>0$, it is guaranteed that there is an available empty slot for a job of length $\ell$ in round $k$. 
Lemma 2. Every schedule minimizing the total flow-time requires at least the same number of migrations as the number of migrations applied by the algorithm.

Proof. We prove the following greedy choice property: for every round $k$ there exists an optimal solution minimizing the total number of migrations, in which the non-migrating jobs assigned to round $k$ are identical to those selected by the algorithm. The following simple observation will be used to analyze the assignment of jobs having lengths in $L_{2}$.

Observation 1 For every round $k$, there are at most two lengths $\ell_{1}, \ell_{2} \in L_{2}$ such that $n_{\ell_{1}, k}>0$ and $n_{\ell_{2}, k}>0$.

Proof. By definition, jobs of lengths in $L_{2}$ span across more than one round in any optimal schedule. Another known property of SPT schedules is that all job lengths in round $k$ are not shorter than job lengths in round $k-1$ and not longer than job lengths in round $k+1$. Therefore, it is not possible to have three different lengths, all spanning over round $k$ and an additional round. In order to preserve the above SPT property, jobs of the middle length, must all be assigned to round $k$.

We prove the greedy choice property for round $k$ : Assume that an optimal schedule agrees with the algorithm in rounds earlier than $k$, and consider the assignment to round $k$. For every machine $i$, if $\operatorname{ForFree}(i, k)=\emptyset$ then this is valid also for the optimal assignment, and a migration from another machine to $S_{i, k}$ is inevitable. If ForFree $(i, k)$ includes at least one job then we use exchange argument to show that any selection of job to $S_{i, k}$ that is different from the algorithm's choice can be changed to the algorithm's choice without hurting the total number of non-migrating jobs. Let $j \in \operatorname{ForFree}(i, k)$ be the job assigned by the algorithm to $S_{i, k}$. Let $j^{\prime} \neq j$ be the job assigned in the optimal schedule to $S_{i, k}$. If $j^{\prime} \notin \operatorname{ForFree}(i, k)$, then by switching $j$ and $j^{\prime}$, we can only reduce the number of non-migrating jobs. If $j^{\prime} \in \operatorname{ForFree}(i, k)$, we distinguish between two cases:

1. $p_{j} \in L_{1}$. In this case, $j$ must be assigned to round $k$, and assigning it to $S_{i, k}$ is the only way to assign it for free. By switching the assignment of $j^{\prime}$ and $j$ in the optimal assignment, we avoid the migration of $j$, and cause a migration to $j^{\prime}$, thus, the total number of migrations does not increase.

2. $p_{j} \in L_{2}$. Since the algorithm gives priority to jobs whose lengths are in $L_{1}$, it must be that all job lengths in $\operatorname{ForFree}(i, k)$ are in $L_{2}$ and in particular, $p_{j^{\prime}} \in$ $L_{2}$. By Observation 1, $p_{j}, p_{j}^{\prime}$ are the only lengths of jobs in ForFree $(i, k)$. Among lengths in $L_{2}$, the algorithm gives priority to shorter jobs, therefore, $p_{j}<p_{j^{\prime}}$. Moreover, $k$ is the last round in which jobs of length $p_{j}$ will be assigned, as otherwise, the SPT order is not preserved (given that jobs of length $p_{j^{\prime}}$ are assigned on both $k$ and $k+1$ ). Therefore, assigning $j$ to $S_{i, k}$ is the only way to assign it for free. By switching the assignment of $j^{\prime}$ and $j$ in the optimal assignment, we avoid the migration of $j$, and cause a migration to $j^{\prime}$, thus, the total number of migrations does not increase. 
We conclude that any optimal assignment can be modified such that it agrees with the algorithm's choice, without hurting the number of migrations. Thus, the algorithm produces an optimal assignment.

Thus, our algorithm produces an optimal schedule using the minimal number of migrations.

Time Complexity Analysis: Algorithm 2 consists of 4 steps. In order to calculate the $n_{\ell, k}$ values in step 1 the jobs should be sorted by processing times. If the initial schedule $S_{0}$ is arbitrary, or if the modification includes jobs addition or jobs' length modification, then the sorting takes in $O(n \log n)$ time. If the initial schedule is optimal, that is, in SPT order, and the modification does not include jobs' length modification, then the algorithm only needs to sort the jobs of each round in $S_{0}$ separately, and concatenate the resulting lists. As there are $m_{0}$ jobs in each round we get an $O\left(n \log m_{0}\right)$ time algorithm. If in the initial SPT schedule the jobs are assigned sequentially on the machines, or if $m_{0}$ is a constant, then Step 1 takes $O(n)$ time.

The partition of job lengths into $L_{1}, L_{2}$ in Step 2 is clearly linear. Step 3 iterates on the rounds and in each round assigns jobs using the already sorted list. The ForFree structure can be implemented using a list of pointers. Since ForFree jobs are assigned in a non-decreasing order and by observation 1, we conclude that this step takes $O\left(m \frac{n}{m}\right)=O(n)$. In step 4, the algorithm assigns the remaining jobs in time $O(n)$.

We conclude that the time complexity of the algorithm varies between $O(n)$ and $O(n \log n)$, depending on the initial schedule and the allowed modification in the instance.

\section{Rescheduling with a Limited Budget - Unit Migration Costs}

In this section we consider the rescheduling problem assuming a limited budget. Naturally, the goal is to utilize the budget in the best possible way, that is, the modified schedule should have a low total flow-time - the minimal possible among all schedules that can be achieved using the given budget. We assume unit migration costs, that is, $\theta_{i i^{\prime} j}=1$, independent of the job $j$ and the involved machines. Thus, the budget $B$ gives the maximal number of allowed migrations. We also assume that $n>B$, as otherwise an optimal schedule can be found by ignoring the migration costs.

The problem can be described as the following weighted matching problem: Similar to the technique used in Section 2.1, let $G=(V, E)$, be a complete bipartite graph with $n$ nodes on one side and $m n$ nodes in the other side. The node $q_{i k}$, for $i=1, \ldots, m$ and $k=1, \ldots, n$, corresponds to the $k^{t h}$ from last position on machine $i$. The edge $\left(j, q_{i k}\right)$ has weight $k p_{j}$, reflecting the contribution of $j$ to the total flow-time if it is assigned on the $k^{\text {th }}$ from last position on machine $i$. We color the edges of $G$ as follows: If an edge $\left(j, q_{i k}\right)$ corresponds to a migration 
of $j$, that is, $i$ is not the machine $j$ is assigned to in $S_{0}$, then the edge is red, otherwise the edge is blue.

It is easy to verify that a min-weight perfect matching with at most $B$ red edges corresponds to an optimal reschedule. For an arbitrary bipartite graph with arbitrary weights, the complexity of the above restricted matching problem is unknown. Some special cases for which efficient algorithms exist include bipartite graphs with unit-weights [15], or with equal sizes $\left(K_{n, n}\right)$ [25. The more general problem of determining whether a complete weighted bipartite graph has a complete matching with a specific weight $w$ in known to be NP-hard [8]. We present optimal polynomial time algorithms for several classes of instances of our problem.

\subsection{The Budget $B$ Is a Constant}

Assume that the modification occurs at time $t=0$, and the budget $B$ is a constant. Clearly, every job $j$ may either migrate or not, and as the budget is a constant, there are at most $n^{B}$ possible ways to select the subset of jobs that are allowed to migrate. The following algorithm considers each selection separately.

Algorithm 3. An optimal algorithm for rescheduling when the budget $B$ is a constant

For every possible selection of $B$ jobs $J^{\prime} \subset J$ :

1. Let $G=(V, E)$, be a bipartite graph with $n$ nodes on one side and $m n$ nodes in the other side. The node $q_{i k}$, for $i=1, \ldots, m$ and $k=1, \ldots, n$, corresponds to the $k^{t h}$ from last position on machine $i$. For every job $j \in J^{\prime}$, there is an edge $\left(j, q_{i k}\right)$ for every $i=1, \ldots, m$ and $k=1, \ldots, n$. For every job $j \notin J^{\prime}$, there is an edge $\left(j, q_{i k}\right)$ for every $k=1, \ldots, n$, but only for the machine $i$ on which $j$ is assigned to in $S_{0}$. The weight of $\left(j, q_{i k}\right)$ is $k p_{j}$.

2. Find a min-cost complete matching in $G$.

Return the schedule induced by the minimal min-cost matching.

Theorem 2. Algorithm 3 returns a modified schedule whose total flow-time is minimal among all schedules achieved with budget at most B.

\subsection{Migrations Are Allowed Only to New Machines}

Another case for which it is possible to solve the problem optimally is when the system's modification consists of machines addition and the only allowed migrations are to the new machines. This scenario arises in practice when the system is upgraded with new machines that are ready to receive tasks, while the old machines are not capable to accept new tasks. We present an optimal algorithm for this problem based on a reduction to a min-cost max-flow problem. An illustration of the flow network is given in Figure 3 . 
An Overview of the Flow Network: The set of nodes $r_{i k}$ for $1 \leq i \leq m_{0}, 1 \leq$ $k \leq n$ correspond to positions on the initial machines. The set of nodes $q_{i^{\prime} k}$ for $1 \leq i^{\prime} \leq m^{\prime}, 1 \leq k \leq B$ correspond to positions on the added machines. All the $q$-nodes are connected to node $d$. The capacity of the edge $(d, t)$ is the budget $B$. This limited capacity guarantees that the total number of slots occupied on the new machines will not exceed $B$. The set of nodes $1 \leq j \leq n$ correspond to the jobs. Every job $j$ that is assigned to machine $i$ in $S_{0}$ is connected to the nodes corresponding to positions on machine $i$ and to all the $q$-nodes. The capacities of all edges except for $(d, t)$ are 1 . The cost of an edge connecting job $j$ to a node corresponding to a $k^{t h}$ from last position (on any machine) is $k p_{j}$. All other edges have cost 0 .

Theorem 3. A minimum-cost maximum-flow (of value $n$ ) in $G$ corresponds to an optimal schedule without exceeding the budget $B$.

Proof. (Sketch) First, note that every valid schedule corresponds to a maximumflow in $G$. On the other hand, not every maximum-flow in $G$ corresponds to a schedule, since a job might be assigned to the $k^{\text {th }}$ from last position in some machine, while less than $k$ jobs are assigned to that machine. However, such a maximum-flow is clearly not of minimal cost - a better matching can be obtained by shifting the $k^{\prime}<k$ jobs assigned to that machine into the $k^{\prime}$ last slots. Therefore, a schedule of minimum total flow-time corresponds to a minimumcost maximum-flow in $G$.

As the capacity of $(d, t)$ is $B$, while all other edges' capacity is 1 , at most $B$ $q$-nodes have incoming flow. These nodes correspond to migrating jobs. Thus, a minimum-cost maximum-flow in $G$ corresponds to an optimal schedule without exceeding the budget $B$.

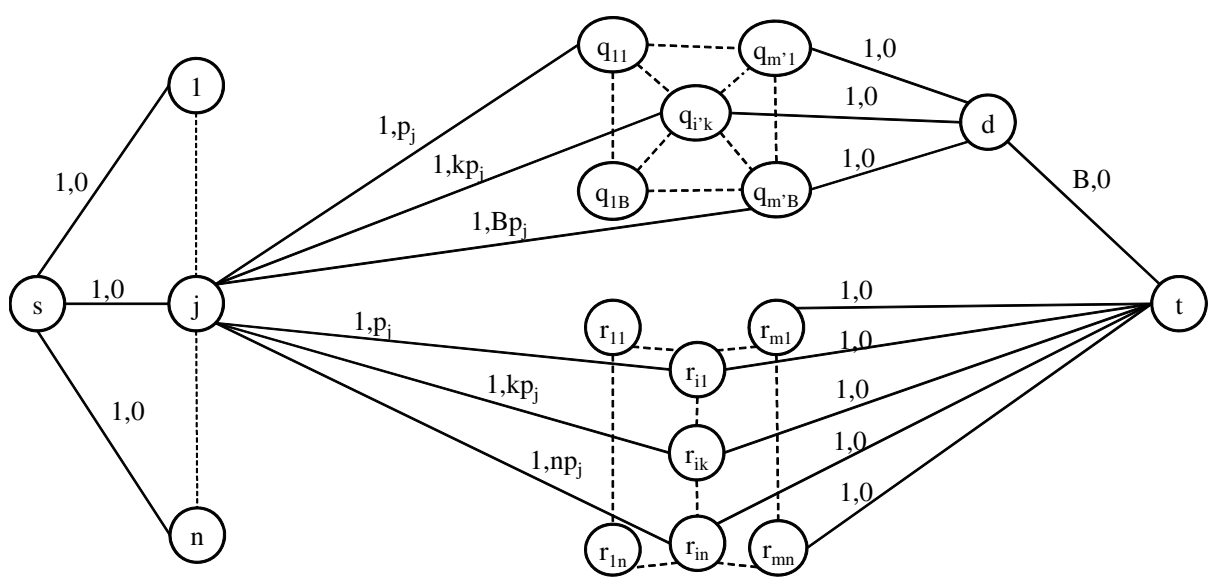

Fig. 3. The flow network built for the rescheduling with limited budget problem. Each edge is labeled by its capacity and the cost of one flow unit. 
This algorithm can be extended for the case in which the systems' modification occurs at time $t>0$ - similar to the extensions described in Section 2.1.1. If restarts are allowed, then our extension assumes that every currently processed job is the shortest job on its machine (which is true if the initial schedule is optimal, or if the schedule is a result of our algorithm - even on a sequence of modifications). If restarts are not allowed then our extension is valid for any initial schedule.

\section{Conclusions and Future Work}

We studied reoptimization problems arising in production planning, in which the goal is to combine the objective of finding a schedule with low total flow-time, with the goal of efficiently converting a given initial schedule to the modified one. We presented the first positive results in this framework. We presented algorithms for finding an optimal schedule achieved using the minimal possible transition cost, and algorithms for optimal utilization of a limited number of migrations.

Several interesting important problems remain open:

1. Identify the complexity status of the second problem for arbitrary transition costs and arbitrary modifications. As explained in Section 3, even with unit transition costs this is a special case of a more general open problem (minweight matching with limited number of red edges).

2. Identify the range of budget $B$ for which it is guaranteed that an optimal reschedule can be achieved using no internal migrations. It is easy to see that this range is included in $m^{\prime}<B \leq m^{\prime} \frac{n}{m_{0}+m^{\prime}}$.

3. Another open research direction is to consider different objective functions. In particular, minimizing the makespan of the schedule, given by the last completion time of some job. Since the problem is NP-hard, the reoptimization problem is clearly also NP-hard. The goal is to develop an algorithm for the reoptimization problem whose approximation-ratio is similar to the best approximation-ratio known for the original problem.

\section{References}

1. Amato, G., Cattaneo, G., Italiano, G.F.: Experimental analysis of dynamic minimum spanning tree algorithms. In: Proc. of 8th SODA (1997)

2. Archetti, C., Bertazzi, L., Speranza, M.G.: Reoptimizing the 0-1 knapsack problem. Discrete Applied Mathematics 158(17) (2010)

3. Ausiello, G., Bonifaci, V., Escoffier, B.: Complexity and approximation in reoptimization. In: Cooper, B., Sorbi, A. (eds.) Computability in Context: Computation and Logic in the Real World. Imperial College Press/World Scientific (2011)

4. Ausiello, G., Escoffier, B., Monnot, J., Paschos, V.T.: Reoptimization of minimum and maximum traveling salesmans tours. J. of Discrete Algorithms 7(4), 453-463 (2009) 
5. Baram, G., Tamir, T.: Reoptimization of the minimum total flow-time scheduling problem (full version), http://www.faculty.idc.ac.il/tami/Papers/BTfull.pdf

6. Böckenhauer, H.J., Forlizzi, L., Hromkovič, J., Kneis, J., Kupke, J., Proietti, G., Widmayer, P.: On the approximability of TSP on local modifications of optimally solved instances. Algorithmic Operations Research 2(2) (2007)

7. Bruno, J.L., Coffman, E.G., Sethi, R.: Scheduling independent tasks to reduce mean finishing time. Communications of the ACM 17, 382-387 (1974)

8. Chandrasekaran, R., Kaboadi, S.N., Murty, K.G.: Some NP-complete problems in linear programming. Operations Research Letters 1, 101-104 (1982)

9. Conway, R.W., Maxwell, W.L., Miller, L.W.: Theory of Scheduling. AddisonWesley (1967)

10. Eppstein, D., Galil, Z., Italiano, G.F.: Dynamic graph algorithms. In: Atallah, M.J. (ed.) CRC Handbook of Algorithms and Theory of Computation, ch. 8 (1999)

11. Escoffier, B., Milanič, M., Paschos, V.T: Simple and fast reoptimizations for the Steiner tree problem. DIMACS Technical Report 2007-01

12. Graham, R.L., Lawler, E.L., Lenstra, J.K., Rinnooy Kan, A.H.G.: Optimization and approximation in deterministic sequencing and scheduling: A survey. Annals of Discrete Math. 5, 287-326 (1979)

13. Grandoni, F., Zenklusen, R.: Optimization with more than one budget. In: Proc. of ESA (2010)

14. Horn, W.: Minimizing average flow-time with parallel machines. Operations Research 21, 846-847 (1973)

15. Karzanov, A.V.: Maximum matching of given weight in complete and complete bipartite graphs. Kibernetika 1, 7-11 (1987); English translation in CYBNAW 23, $8-13$

16. Mattox, D.: Handbook of Physical Vapor Deposition (PVD) Processing, 2nd edn. Elsevier (2010)

17. Nardelli, E., Proietti, G., Widmayer, P.: Swapping a failing edge of a single source shortest paths tree is good and fast. Algorithmica 35 (2003)

18. Pallottino, S., Scutella, M.G.: A new algorithm for reoptimizing shortest paths when the arc costs change. Operations Research Letters 31 (2003)

19. Ravi, R., Goemans, M.X.: The Constrained Minimum Spanning Tree Problem. In: Karlsson, R., Lingas, A. (eds.) SWAT 1996. LNCS, vol. 1097, pp. 66-75. Springer, Heidelberg (1996)

20. Shachnai, H., Tamir, G., Tamir, T.: Minimal Cost Reconfiguration of Data Placement in Storage Area Network. In: Bampis, E., Jansen, K. (eds.) WAOA 2009. LNCS, vol. 5893, pp. 229-241. Springer, Heidelberg (2010)

21. Shachnai, H., Tamir, G., Tamir, T.: A Theory and Algorithms for Combinatorial Reoptimization. In: Fernández-Baca, D. (ed.) LATIN 2012. LNCS, vol. 7256, pp. 618-630. Springer, Heidelberg (2012)

22. Sitters, R.A.: Two NP-Hardness Results for Preemptive Minsum Scheduling of Unrelated Parallel Machines. In: Aardal, K., Gerards, B. (eds.) IPCO 2001. LNCS, vol. 2081, pp. 396-405. Springer, Heidelberg (2001)

23. Smith, W.E.: Various optimizers for single-stage production. Naval Research Logistics Quarterly 3, 59-66 (1956)

24. Thorup, M., Karger, D.R.: Dynamic Graph Algorithms with Applications. In: Halldórsson, M.M. (ed.) SWAT 2000. LNCS, vol. 1851, pp. 1-9. Springer, Heidelberg (2000)

25. Yi, T., Murty, K.G., Spera, C.: Matchings in colored bipartite networks. Discrete Applied Mathematics 121, 261-277 (2002) 